\title{
Ressonância Nuclear Magnética no Diagnóstico de Pseudoaneurisma de Ventrículo Esquerdo. Antigo Problema, Nova Opção
}

\author{
Glaura S. Alvarenga, Liliane Kopel, Luís A lberto O liveira Dallan, Luiz Francisco Avila, \\ Maristela C. Monachini, Silvia G. Lage
}

São Paulo, SP

Paciente feminina, 67 anos, internada por infarto agudo do miocárdio de parede lateral, com edema agudo de pulmão e evolução para choque cardiogênico nas primeiras horas. Ecocardiograma transesofágico e ressonância nuclear magnética confirmaram o diagnóstico de pseudoaneurisma de ventrículo esquerdo. A paciente foi submetida a tratamento cirúrgico com sucesso.

\section{Nuclear Magnetic Resonance Imaging in the Diagnosis of Left Ventricular Pseudoaneurysm. An Old Problem, a New Option}

We describe the case of a 61 year-old female patient admitted to the hospital with acute lateral myocardial infarction, in pulmonary edema, who evolved to cardiogenic shock in the first hours. Transesophageal echodopplercardiogram and nuclear magnetic resonance imaging showed the diagnosis of a left ventricular pseudoaneurysm. Surgical repair was successfully undertaken.

Arq Bras Cardiol, volume 69 (n 4), 251-254, 1997

Pseudoaneurisma de ventrículo esquerdo (VE) é o resultado de uma rotura do miocárdio, na qual o hematoma resultante está contido em uma área circunscrita pelo pericárdio. Assim, os pseudoaneurismas têm sua parede formada por pericárdio e tecidos extracardíacos, enquanto nos aneurismas verdadeiros a parede é formada por miocárdio necrosado e tecido fibroso. Os pseudoaneurismas formam uma cavidade conectada ao VE por um colo estreito e podem apresentar trombose em seu interior. As causas mais freqüentemente associadas à formação de pseudoaneurisma de VE são infarto agudo do miocárdio (IAM), complicações de procedimentos cirúrgicos, trauma torácico ou endocardite bacteriana ${ }^{1,2}$.

Apesar das manifestações clínicas dos pseudoaneurismas serem inespecíficas, o diagnóstico preciso é importante para o tratamento adequado, sendo que o uso de métodos por imagem desempenha um papel fundamental ${ }^{3}$.

O diagnóstico precoce e a correção cirúrgica são importantes para a diminuição da mortalidade e a correção ci-

Instituto do Coração do Hospital das Clínicas - FMUSP

Corespondência: Glaura S. Alvarenga - Rua Josemar Moreira de Mello, 196 - 04114 040 - São Paulo, SP

Recebido para publicação em 9/10/96

Aceito em 20/8/97 rúrgica deve ser feita independente da presença de sintomas ou da necessidade de revascularização do miocárdio concomitante ${ }^{4,5}$.

Apresentamos o caso de um portador de pseudoaneurisma de VE pós-IAM da parede lateral, em que métodos de imagem não-invasivos, isto é, ecocardiograma transesofágico e ressonância nuclear magnética foram decisivos para o diagnóstico. Dois meses após o infarto, o paciente foi submetido à correção cirúrgica com sucesso.

\section{Relato do Caso}

Mulher de 61 anos, com antecedente de infarto do miocárdio ínfero-látero-dorsal em agosto/95 e submetida a desobstrução mecânica e angioplastia transluminal coronária da artéria circunflexa na fase aguda do infarto.

Em 23/3/96, deu entrada no serviço de emergência com IAM em edema agudo do pulmão. Na evolução apresentou choque cardiogênico, taquicardia ventricular, bloqueio atrioventricular total intermitente, bloqueio de ramo direito e bloqueio divisional ântero-superior do ramo esquerdo, sendo necessário implante de marcapasso provisório.

Indicado cateterismo cardíaco com cineangiocoronariografia de urgência que revelou: artéria circunflexa ocluída, artéria descendente anterior com $70 \%$ de obstru- 
ção, artéria coronária direita com $95 \%$ de obstrução e artéria ventricular posterior com $80 \%$ de obstrução. Efetuada desobstrução mecânica e angioplastia da artéria circunflexa com sucesso.

Evoluiu com quadro de choque séptico, com provável foco infeccioso primário pulmonar, necessitando drogas vasopressoras por 11 dias e inotrópicos por 30 dias, além de antibioticoterapia de amplo espectro. Apresentou, como complicações: insuficiência renal aguda revertida e acidente vascular cerebral isquêmico parieto-occiptal esquerdo, confirmado por tomografia computadorizada de crânio.

No $11^{\circ}$ dia após o infarto do miocárdio, foi realizado ECO transtorácico que sugeriu a presença de um pseudoaneurisma em parede ínfero-lateral adjacente ao músculo papilar póstero-medial, melhor evidenciado pelo ecocardiograma transesofágico realizado a seguir. A ressonância nuclear magnética confirmou o diagnóstico de pseudoaneurisma de VE(fig. 1A).

Em vista da estabilização do quadro hemodinâmico e infeccioso, 58 dias após o IAM, foi indicada a correção cirúrgica do pesudoaneurisma e revascularização do miocárdio. O achado macroscópico intra-operatório confirmou o diagnóstico prévio. Procedeu-se a reconstrução da parede ven-
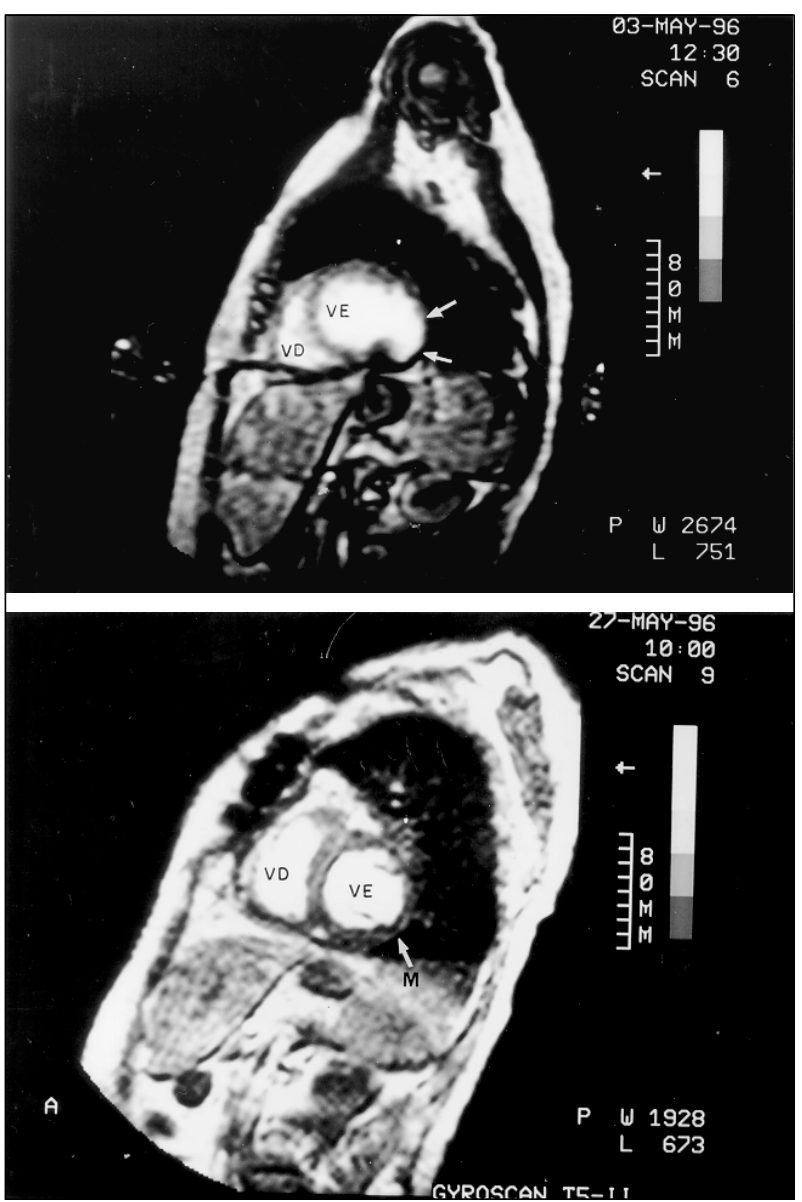

Fig. 1 - Ressonância nuclear magnética evidenciando as cavidades ventriculares direita (VD) e esquerda (VE). A) pseudoaneurisma de VE (setas) havendo nítida descontinuidade da parede miocárdica; B) parede miocárdica (M) íntegra após reconstrução cirúrgica. tricular após retirada dos trombos e realizou-se a revascularização da artéria descendente anterior e da $1^{\mathrm{a}}$ marginal esquerda (fig. 2). A ressonância nuclear magnética realizada no $8^{\circ}$ pós-operatório evidenciou o sucesso cirúrgico da correção do pseudoaneurisma (fig. 1B). A paciente recebeu alta 70 dias após o IAM.

\section{Discussão}

Os pseudoaneurismas de VE apresentam maior risco de complicações quando comparados aos aneurismas verdadeiros, sendo a mais grave a rotura espontânea, pois pode levar à morte súbita ${ }^{4,5}$. A rotura precoce, mais comum do que a tardia, ocorre ao redor da $5^{\mathrm{a}}$ semana após o infarto, antes da formação do tecido fibroso ${ }^{6,7}$. A rotura é responsável por 5 a $8 \%$ das mortes nas primeiras semanas após IAM e ocorre, preferencialmente, na parede anterior do $\mathrm{VE}^{4}$. A manifestação clínica mais comum dos pseudoaneurismas de VEé a insuficiência cardíaca congestiva, seguida da angina de peito. Síncope, complicações tromboembólicas, arritmias ventriculares e endocardite são extremamente ra$\operatorname{ras}^{8}$.

Em relação à topografia, os pseudoaneurismas, usualmente, são encontrados na parede inferior, posterior ou lateral, em contraste com os aneurismas verdadeiros que têm distribuição tipicamente ântero-apical ${ }^{9,10}$. Pseudoaneurismas de parede posterior são 3,5 vezes mais comuns do que de parede anterior. Entretanto, a rotura aguda é mais comum nos pseudoaneurismas de parede anterior ${ }^{8}$. Em revisão de 71 casos de pseudoaneurismas após IAM, 74\% localizavam-se na parede inferior do VE. A artéria coronária direita foi acometida de forma isolada em $72 \%$ dos casos relatados e em $28 \%$ havia comprometimento bi ou triarterial ${ }^{9}$.

O diagnóstico definitivo do pseudoaneurisma é anatomopatológico, visto que a ausência de fibras miocárdicas em suas paredes permite diferenciação dos aneurismas verdadeiros $^{3}$.

O diagnóstico clínico dos pseudoaneurismas é difícil, sendo muitas vezes um achado cirúrgico ou de necropsia. $\mathrm{O}$ exame físico, a radiografia de tórax e o eletrocardiograma são pouco sensíveis para a detecção dos pseudoaneurismas ${ }^{3}$. Exames complementares, como ecodoppplercardiograma, ventriculografia radioisotópica, tomografia computadorizada, ressonância nuclear magnética ou cateterismo cardíaco com ventriculografia esquerda, muitas vezes, podem ser necessários para a definição diagnóstica. Assim, os exames complementares não-invasivos, em particular o ecocardiograma transesofágico e a ressonância nuclear magnética têm destaque especial por serem sensíveis e específicos.

A realização da ecocardiografia bidimensional transtorácica pode demonstrar interrupção brusca do endocárdio, câmara sacular paraventricular e colo de união do pseudoaneurismaao VE. Odiâmetro máximo docoloé sempre inferior ao diâmetro máximo da cavidade do pseudoaneurisma ${ }^{11}$. A presença de colo, como característica típica dos pseudoaneurismas, tem sido questionada, pois vem sendo relatada nos aneurismas verdadeiros ${ }^{12,13}$. A ecocardiografia permite 
ainda a visibilização direta de trombos no interior dos pseudoaneurismas ${ }^{3}$. O Doppler pulsátil permite a detecção de fluxo sistólico e diastólico no colo do pseudoaneurisma, o que confirma a comunicação entre as duas câmaras ${ }^{14}$.

Alguns pseudoaneurismas inferiores podem passar desapercebidos ao ecocardiograma transtorácico. Nestes casos, a ventriculografia isotópicaé um método melhor para o diagnóstico ${ }^{3,15}$. A presença de uma cavidade extracardíaca sugere um pseudoaneurisma ${ }^{3}$. O ecocardiograma transesofágico é superior na documentação de pseudoaneurismas posteriores ${ }^{16,17}$.

Com o advento da tomografia e ressonância nuclear magnética surgiu a possibilidade de definir com precisão o contorno cardíaco de forma não-invasiva. Para que o diagnóstico seja estabelecido com o uso da tomografia e da ressonância nuclear magnética é necessária a demonstração de rotura da gordura epicárdica e adesões intrapericárdicas. A presença de gordura epicárdica íntegra adjacente a um aneurisma, exclui a possibilidade de um pseudoaneurisma ${ }^{18}$.

A tomografia contrastada visibiliza claramente imagens intra e extracardíacas, auxiliando no diagnóstico diferencial entre derrame pericárdico, hematoma intrapericárdico e cisto de pericárdio ${ }^{18}$.

Em contraste com a tomografia, a comunicação entre o pseudoaneurisma e o VE necessita ser analisada pela ressonância nuclear magnética, para excluir o diagnóstico de outras cavidades intrapericárdicas, porém extracardíacas ${ }^{18}$.

A cine-ressonância nuclear magnética, usando a técnica de eco-gradiente, é um método que foi primeiro utilizado para quantificar a função regional e global do coração. Esta técnica provou sua eficácia em identificar precisamente a localização regional e quantificar a função do miocárdio infartado, particularmente, quando este foi reperfundido precocemente. Assim, cine-ressonância magnética, devido a sua alta sensibilidade em detectar alterações de fluxo, é recomendada nos casos de suspeita de rotura do miocárdio. Este método parece ser o único capaz de mostrar a rotura do miocárdio precoce, decorrente de IAM, impossível de ser detectada pelos outros métodos, inclusive a spin-eco-ressonância nuclear magnética ${ }^{19}$.

Desta forma, quando houver suspeita do diagnóstico de pseudoaneurisma de VE, em vista de alta morbidade e mortalidade, a realização de investigação não-invasiva deve ser precoce, visando o adequado tratamento cirúrgico. $\mathrm{O}$ método de investigação diagnóstico a ser escolhido dependerá da disponibilidade do serviço, da experiência pessoal, do custo e das condições clínicas do paciente. A ecocardiografia transtorácica ou transesofágica é sensível, apesar da

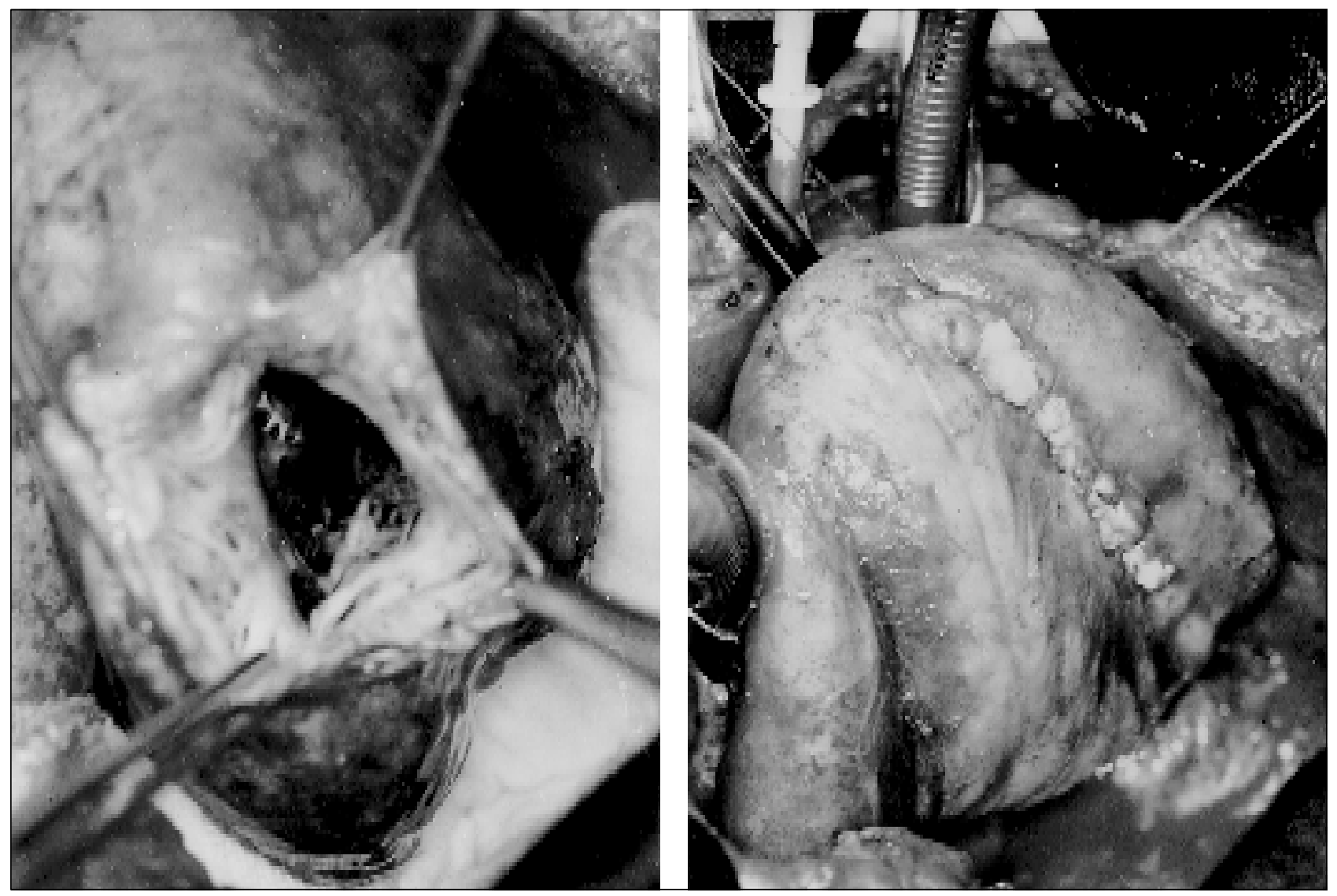

Fig. 2 - A) Aspecto macroscópico da rotura da parede pósteo-lateral do VE, já liberada do pericárdio. O coração encontra-se luxado sobre a sua face anterior e os trombos foram retirados. Pode-se visibilizar o músculo papilar posterior da valva mitral, que foi preservado, e o preparo das bordas remanescentes viáveis desse ventrículo, para sua reconstrução geométrica; B) aspecto final da parede póstero-lateral do VE (a ponta do coração está luxada anteriormente), após correção da rotura, ressecção do pseudoaneurisma e sutura primária das paredes remanescentes do VE. 
sua limitação conforme a localização dos pseudoaneurismas. É um método de menor custo, possível de ser realizado à beira leito nos pacientes mais graves. A ressonância nuclear magnética é um método não-invasivo, sensível e específico, embora apresente alto custo e necessidade de estabilidade clínica do paciente.

\section{Referências}

1. Roberts WC, Morrow AG - Pseudoaneurysm of the left ventricle. An unusual sequel of myocardial infarction and rupture of the heart. Am J Med 1967; 43: 639-44

2. Dachman AH, Spindola-Franco H, Solomon N - Left ventricula pseudoaneurysm. Its recognition and significance. JAMA 1981; 246: 1951-3.

3. Riera JC, Daví AR, Bruix AS et al - Diagnóstico de los seudoaneurismas ventriculares. Papel complementario de la ecocardiografía, la ventriculografía istópica y la ventriculografía de contraste. Rev Esp Cardiol 1993; 46: 225-34.

4. VlodaverZ, Coe JI, Edwards JE-True and false left ventricular aneurysm: Propensity for the latter rupture. Circulation 1975; 51: 567-72.

5. Van Tassel RA, Edwards JE - Rupture of heart complicating myocardial infarction: analysis of 40 cases including nine examples of left ventricular false aneurysm. Chest 1972; 61: 104-16.

6. Otto CM, Stratton JR - Postinfarction left ventricular pseudoaneurysm: echocardiographic diagnosis and prolonged survival in three patients. Clin Cardiol 1988; 11: 189-92.

7. Passos LCS, Oliveira SF, Ramires JAF, Jatene F, Jatene A - Pseudoaneurisma do ventrículo esquerdo ressecado vinte anos após infarto agudo do miocárdio. Arq Bras Cardiol 1991; 57: 479-81.

8. Komeda M, David TE-Surgical treatment of post infarction false aneurysm of the left ventricle. J Thorac Cardiovasc Surg 1993; 106: 1189-91.

9. Milgarter E, Uretzky G, Levy P, Appelbaum A, Borman B - Pseudoaneurysm of left ventricle. Thorac Cardiovasc Surgeon 1987; 35: 20-5.

10. March KL, Swada SG, Tarver RD, Kesler KA, Armstrong WF-Current concept of left ventricular pseudoaneurysm: pathophysiology, therapy, and diagnostic imaging methods. Clin Cardiol 1989; 12: 531-40.
11. Gatewood RP, Nanda NC - Differentiation of left ventricular pseudoaneurysm from true aneurysm with two dimensional echocardiography. Am J Cardiol 1980; 46: 869-78.

12. Davies MJ - Ischaemic ventricular aneurysms: true or false? Br Heart J 1988; 60 : 95-7.

13. Lascault G, Reeves F, Drobinski G - Evidence of the inaccuracy of standard echocardiographic and angiographic criteria used for the recognition of true and "false" left ventricular inferior aneurysms. Br Heart J 1988; 60: 125-7.

14. GascóFJC, Mas MLM, Merino VL, Casares AL, Sesma JM-Seudoaneurisma ventricular izquierdo. Dinámica y patrones de flujo analizados mediante ecocardiografía - Doppler. Rev Esp Cardiol 1991; 44: 269-72.

15. Sutherland GR, Smyllie JH, Roelandt JRTC - Advantages of colour flow imaging in the diagnosis of left ventricular pseudoaneurysm. Br Heart J 1989; 61: 59-64.

16. Fehske W, Kranidis A, Kirchhoff PG, Omran H, Lauck G, Lüderitz B - Diagnosis of a posterior left ventricular pseudoaneurysm by multiplane transesophageal echocardiography. J Clin Ultrasound 1995; 23: 59-62.

17. Stoddard MF, Dawkins PR, Longaker RA, Goad J, Shih A - Transesophageal echocardiography in the detection of left ventricular pseudoaneurysm. Am Heart J 1993; 125: 534-9.

18. Duvernoy O, Wikström G, Mannting F, Larsson SG, Andrén B, Dubiel T - Pre and postoperative $\mathrm{CT}$ and MR in pseudoaneurysms of the heart. J Comput AssitTomogr 1992; 16: 401-9.

19. Chatelain P, Didier D - Detection of myocardial rupture by cine-magnetic resonance imaging. Am J Cardiol 1994; 73: 1033-5. 